\title{
The Same Antidepressant Elicits Contrasting Patterns of Synaptic Changes in the Amygdala vs Hippocampus
}

\author{
Anup Gopalakrishna Pillai', Shobha Anilkumar' and Sumantra Chattarji*, I \\ 'National Centre for Biological Sciences, Bangalore, India
}

As depression-like symptoms are often precipitated by some form of stress, animal models of stress have been used extensively to investigate cellular mechanisms of depression. Despite being implicated in the emotional symptoms of depression, the amygdala has received little attention compared to the hippocampus in the past studies of antidepressant action. Further, these investigations have not taken into account the contrasting effects of chronic stress on the hippocampus vs amygdala. If an antidepressant is to be equally effective in countering the differential effects of stress on both brain areas, then it is faced with the challenge of eliciting contrasting effects in these two structures. We tested this prediction by examining the impact of tianeptine, an antidepressant with proven clinical efficacy, on neurons of the lateral amygdala (LA) and hippocampal area CAI. Tianeptine reduces N-methyl-D-aspartate (NMDA)-receptor-mediated synaptic currents, without affecting $\alpha$-amino-3-hydroxy-5-methyl-4-isoxazole propionate (AMPA) currents, in LA neurons. By contrast, tianeptine enhances both NMDA and AMPA currents in area CAI. Tianeptine also lowers action potential firing in LA neurons. As tianeptine modulates cellular metrics that, in addition to mediating amygdalar behavioral output, are also affected by stress, we tested if tianeptine succeeds in countering stress effects in the intact animal. We find that tianeptine prevents two important functional consequences of chronic stress-induced plasticity in the amygdala — dendritic growth and enhanced anxiety-like behavior. These results provide evidence for antidepressant action on amygdalar neurons that are not only distinct from the hippocampus, but also protect against the debilitating impact of stress on amygdalar structure and function.

Neuropsychopharmacology (2012) 37, 2702-27II; doi:I0.1038/npp.20I2.135; published online 25 July 2012

Keywords: amygdala; excitability; dendritic remodeling; NMDA; AMPA; stress

\section{INTRODUCTION}

Research on the neurobiology of depression and antidepressant action is faced with the challenge of elucidating the complex roles of genetic and environmental factors underlying the pathophysiology of the disorder. This in turn has impeded the development of animal models that fully capture the range of symptoms seen in the human condition (Nestler et al, 2002). Despite these limitations, two lines of investigation have been particularly fruitful. First, animal models have utilized antidepressant treatments that are effective in alleviating depressive symptoms in humans. Second, studies have also focused on the striking parallels between key features of severe depression and dysregulation of the stress response. Abnormally high activation of the hypothalamic-pituitary-adrenal (HPA) axis is reported in almost half of the individuals afflicted with depression, and antidepressant treatment is able to reverse many of these

* Correspondence: Professor S Chattarji, National Centre for Biological Sciences, GKVK, Bellary Road, Bangalore 560065, India, Tel: +91 80 23666|20, Fax: +9I 80 23636662, E-mail: shona@ncbs.res.in

Received 20 February 2012; revised 22 June 2012; accepted 29 June 2012 aberrations (Arborelius et al, 1999; Nestler et al, 2002; Sachar and Baron, 1979). Further, neuroimaging studies have reported a loss of volume in the hippocampus, which regulates the stress response via the HPA axis (Bremner et al, 2000; Frodl et al, 2002). Importantly, these symptoms of depression are captured in rodent models of repeated stress that cause dendritic atrophy in the hippocampus, which too can be corrected with certain antidepressants (Magarinos et al, 1999; McEwen et al, 2002; Watanabe et al, 1992). Taken together, these studies have given rise to models of stress-induced impairment of hippocampal function that are believed to underlie many of the neuroendocrine and cognitive symptoms of depression (McEwen, 1999).

The intellectual framework emerging from studies on the hippocampus, however, is unlikely to account for all of the affective symptoms of depression. Although symptoms of anxiety and fear are prominent in many depressed individuals, the amygdala has received relatively little attention in depression research. Indeed, little is known about how antidepressants affect amygdalar neurons. The need to look beyond the hippocampus is also highlighted by growing evidence that rodent models of stress elicit contrasting patterns of structural plasticity in the hippocampus $v s$ 
amygdala (Kavushansky et al, 2006; Roozendaal et al, 2009; Vouimba et al, 2004). For example, the same chronic stress that triggers dendritic atrophy in the hippocampus causes dendritic growth in the basolateral amygdala (BLA) (McEwen, 1999; Vyas et al, 2002). These reports pose a significant challenge to therapeutic interventions aimed at correcting the functional consequences of contrasting patterns of stressinduced plasticity in the hippocampus $v s$ amygdala. In other words, to be effective across both of these brain regions, an antidepressant has to be equally adept at selectively countering these specific, and often opposite, cellular changes. Here we explored this possibility by using tianeptine, an antidepressant with proven clinical efficacy that has been reported to block many of the molecular, cellular, and behavioral effects of chronic stress in the hippocampus (Kim et al, 2006; McEwen et al, 2010). Despite possessing a heterocyclic structure, tianeptine is chemically dissimilar to tricyclic agents and does not inhibit the uptake of serotonin in the central nervous system (McEwen et al, 2010). Although earlier findings suggested that tianeptine enhances serotonin reuptake, recent measurements based on more reliable techniques have shown that administration of tianeptine does not elicit any marked changes in extracellular levels of serotonin. Instead, converging lines of evidence demonstrate that the antidepressant actions of tianeptine can be attributed to its modulation of the glutamatergic system (McEwen et al, 2010). Therefore, in this study we first examined if tianeptine triggered distinct electrophysiological effects in single neurons of the amygdala $v s$ hippocampus. We then investigated if the physiological changes elicited by tianeptine in the amygdala have functional consequences for the impact of stress on its structure and behavioral output.

\section{MATERIALS AND METHODS}

\section{Animals}

Male Wistar rats (45-60 days old, 250-300 g) were housed in a 14/10 h light/dark schedule (lights on at 0800 hours) with ad libitum access to food and water at the National Centre for Biological Sciences (NCBS), Bangalore, India. The NCBS Institutional Animal Ethics Committee approved all procedures related to animal maintenance/experimentation.

\section{Slice Electrophysiology}

Naïve animals, after anesthesia, were decapitated and the brain was dissected, sliced $(400 \mu \mathrm{m})$, and stored in oxygenated $\left(95 \% \mathrm{O}_{2}, 5 \% \mathrm{CO}_{2}\right)$ artificial cerebrospinal fluid (ACSF) at room temperature. Whole-cell current and voltage-clamp recordings from projection neurons were obtained in the dorsal lateral amygdala (LA) and hippocampal area CA1 using previously established procedures (Suvrathan et al, 2010). Briefly, stimulation of afferent fibers from the internal capsule, containing thalamic afferents (to LA), and Schaffer collateral inputs to CA1 elicited monosynaptic excitatory postsynaptic currents (EPSCs), which were recorded using an EPC-9 amplifier (HEKA Elektronik, Lambrecht, Germany). Further details are available in Supplementary Information.

\section{Behavioral and Pharmacological Procedures}

Chronic immobilization stress (CIS) consisted of containment (with no access to food or water) in rodent immobilization bags for $2 \mathrm{~h}$ (1000-1200 hours) for 10 consecutive days (Vyas et al, 2002). Rodent immobilization bags are cone-shaped polyethene bags with the apex of the cone cut open. Each rat was placed within the bag with the nose emerging from the cut end, allowing unrestricted breathing. The open rear end (ie, the base of the cone-shaped bag) was then closed with a tape, such that the rat was immobilized within the bag. Experimenters ensured that the rat's legs were not folded and the neck was not bent abnormally. After stress, the animals were returned to home cages and subjected to behavioral/morphological analysis 1 day after chronic stress. Animals were randomly assigned to either stress or control (unstressed) groups. Half the animals in each group received tianeptine $(10 \mathrm{mg} / \mathrm{kg}$, intraperitoneally), whereas the other half received vehicle (sterile $0.9 \% \mathrm{NaCl}$, intraperitoneally) injections. Vehicle/tianeptine injections were given $30 \mathrm{~min}$ before the start of daily stress. Tianeptine sodium salt $\left(\mathrm{C}_{21} \mathrm{H}_{24} \mathrm{ClN}_{2} \mathrm{NaO}_{4} \mathrm{~S}\right)$ was provided by Servier (Nanterre, France).

\section{Behavioral Measurements}

Anxiety-like behavior was assessed in the elevated plus maze. The maze consisted of two opposite open arms $\left(60 \times 15 \mathrm{~cm}^{2}\right.$, surrounded by $1-\mathrm{cm}$-high transparent wall) and two opposite enclosed arms $\left(60 \times 15 \mathrm{~cm}^{2}\right.$, surrounded by a $15-\mathrm{cm}$-high opaque wall) perpendicular to each other and elevated $75 \mathrm{~cm}$ from ground. Individual trials, lasting for $5 \mathrm{~min}$, were videotaped for off-line analysis. Animals were placed at the center of the maze facing an enclosed arm and the maze was cleaned with $5 \%$ ethanol solution $(\mathrm{v} / \mathrm{v})$ after each trial. Animal were tested (1000-1400 hours) 1 day after the 10th day of chronic stress.

\section{Golgi Staining and Morphological Analysis}

One day after the end of stress, animals were anesthetized and decapitated after testing on the plus maze. Brains were quickly removed and processed for rapid Golgi staining (Ramkumar et al, 2008). Dendritic arbors of Golgi-impregnated BLA neurons were quantified using Sholl's analysis as described earlier (Vyas et al, 2002; Supplementary Information).

\section{Statistical Analysis}

Comparisons between groups (control $v s$ tianeptine) were performed on electrophysiological data collected at identical time points using independent $t$-test. Paired $t$-test was used to compare values at different epochs (drug/washout) with the respective baseline. Significances for cumulative distributions were obtained from two-sample KolmogorovSmirnov (K-S) test. Morphological and behavioral data were analyzed with one-way ANOVA, followed by post-hoc (Tukey's) tests. Additional analysis of behavioral data was carried out with a more rigorous two-way ANOVA to assess the main effects of drug (tianeptine $v s$ saline), treatment (stress $v s$ control), and interactions (drug vs treatment). Normality of data was tested using one-sample K-S test. 
Homogeneity of variance was tested with Levene's test. SPSS 9.0 was used for all statistical analyses.

\section{RESULTS}

\section{Tianeptine Potentiates Synaptic Currents Mediated by AMPA and NMDA Receptors in the Hippocampus}

Stress has adverse effects on hippocampal structure and function at multiple levels of neural organization. The atypical antidepressant tianeptine is reported to be remarkably effective in preventing many of these stress effects on structural and physiological markers of plasticity, including those involving glutamatergic transmission (Kole et al, 2002; Qi et al, 2009; Reagan et al, 2004; Reznikov et al, 2007; Vouimba et al, 2006; Watanabe et al, 1992). Therefore, we first investigated the effects of tianeptine on EPSCs mediated by $\alpha$-amino-3-hydroxy-5-methyl-4-isoxazole propionate receptors (AMPARs) and $N$-methyl-D-aspartate receptors (NMDARs).
Whole-cell voltage-clamp recordings of AMPAR-EPSCs, evoked by stimulation of Schaffer collateral inputs, were obtained from CA1 pyramidal neurons in hippocampal slices (Figure 1a; Supplementary Information). Bath application of tianeptine $(50 \mu \mathrm{M}, 15 \mathrm{~min})$ caused a robust increase in the AMPA-EPSC peak amplitude, which gradually declined near baseline level by the end of a 30-min washout period (Figure 1a). As depicted in the time-course plot averaged across all experiments (Figure 1a, bottom), at the end of the 15-min period of drug application, the AMPAEPSC peak amplitude was significantly greater than the predrug baseline value. However, this potentiating effect did not persist at the same level after ACSF washout. None of the above effects were observed in control experiments with ACSF application (Figure 1a, bottom, open circles).

The relative impact of tianeptine, followed by its washout, on AMPA-EPSCs in all CA1 cells recorded is shown as cumulative probability plots (Figure $1 \mathrm{~b}$ ). There was a significant rightward shift (ie, increase in AMPA-EPSC peak amplitude; $p=0.013$ ) in all cells exposed to $15 \mathrm{~min}$ of

a

b
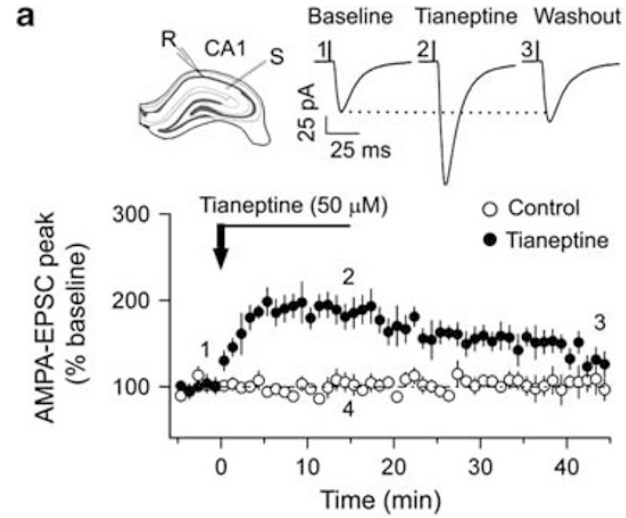

c

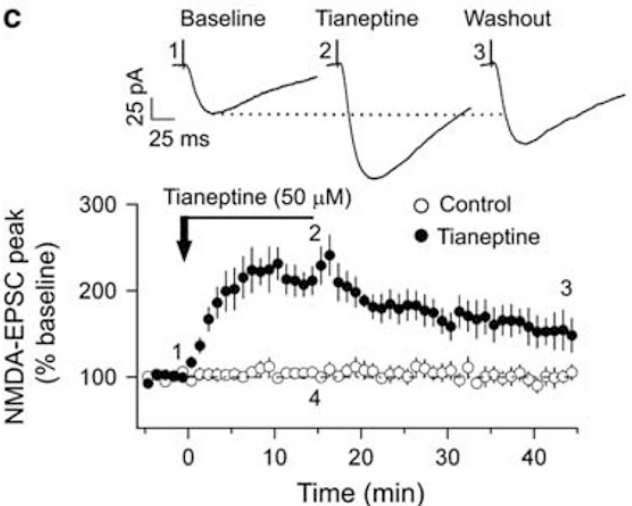

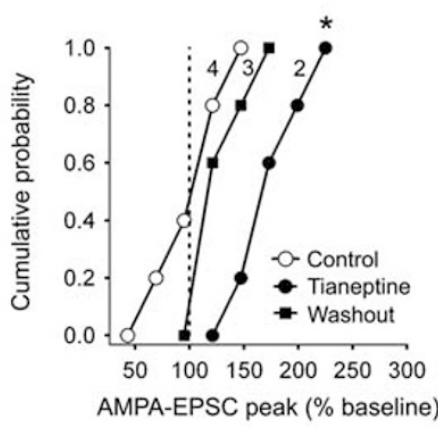

d

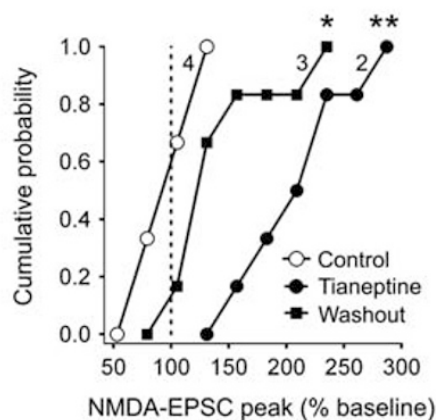

Figure I Tianeptine enhances glutamatergic currents in CAI pyramidal neurons. (a, top left) Schematic of the hippocampal slice preparation with recording (R) and stimulation (S) electrodes. (Top right) Representative $\alpha$-amino-3-hydroxy-5-methyl-4-isoxazole propionate receptors-excitatory postsynaptic currents (AMPAR-EPSCs) during baseline, at the end of tianeptine (50 $\mu \mathrm{M}, 15 \mathrm{~min}$ ), and washout (artificial cerebrospinal fluid (ACSF), 30 min). (Bottom) Time-course plot of AMPA-EPSCs indicating the increase in peak response after tianeptine application $(189 \pm 14 \%, n=5, p=0.0005)$ and washout $(135 \pm 10 \%, n=5, p=0.1 \mathrm{I})$ compared to the steady responses of control neurons $(n=6)$ that received ACSF application. (b) Cumulative probability density plot indicating the significant $(* 2<0.02)$ rightward shift of peak AMPA currents at the end of tianeptine application in comparison to the pre-drug baseline (dotted lines) and control cells. (c, top) Representative traces of N-methyl-D-aspartate receptor (NMDAR)-EPSCs during baseline, at the end of tianeptine application, and washout. (Bottom) The time-course plot depicts the increase in NMDA-EPSC amplitude by tianeptine, both during application ( $216 \pm 17 \%, n=6, p=0.0009)$ and washout ( $153 \pm 20 \%, p=0.029)$, while control neurons $(n=6)$ were not affected. (d) Cumulative probability density of NMDA-EPSCs exhibit a significant (** $<0.005)$ rightward shift with tianeptine application, which was also persistent $(* p<0.03)$ during washout compared to control cells or pre-drug baseline (dotted lines) levels. The epoch of sample traces and cumulative plots are indicated by the position of the respective markers on the time-course plot. Significances were obtained by comparing tianeptine and control neurons at similar time points (as indicated by the markers) using two-sample Kolmogorov-Smirnov test. Error bars represent standard error of mean (SEM). 
a
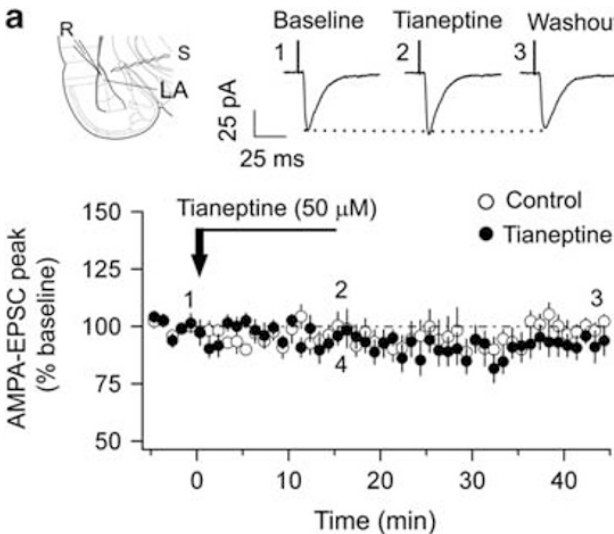

C
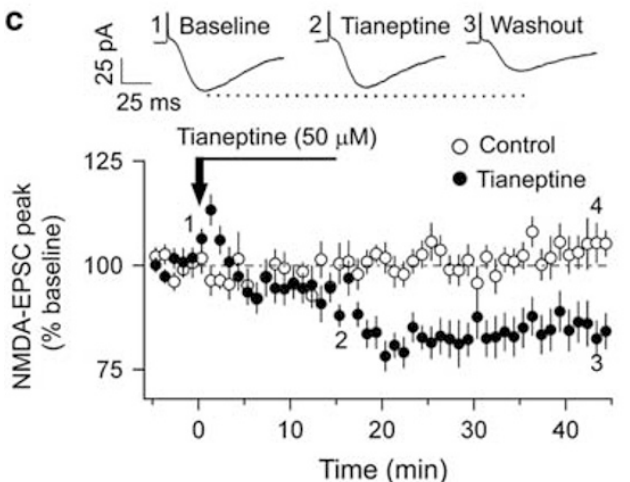

b

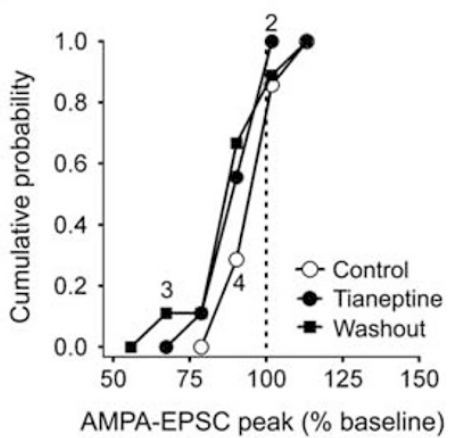

d

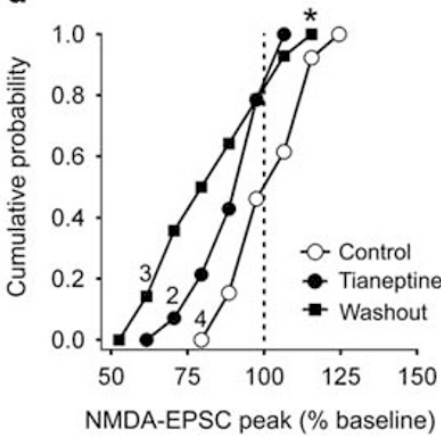

Figure 2 Tianeptine reduces N-methyl-D-aspartate receptor (NMDAR)-mediated excitatory postsynaptic current (EPSCs) in lateral amygdala (LA) neurons without altering $\alpha$-amino-3-hydroxy-5-methyl-4-isoxazole propionate receptor (AMPAR)-mediated currents. (a, top left) Schematic of the amygdalar slice preparation with recording $(R)$ and stimulation $(S)$ electrodes. (Top right) Representative AMPAR-EPSCs during baseline, at the end of tianeptine $(50 \mu \mathrm{M}, 15 \mathrm{~min})$ application, and washout. (Bottom) The time-course plot of AMPA-EPSCs were similar between tianeptine-applied cells (Tia: $94 \pm 3 \%, p=0.44$; washout: $93 \pm 5 \%, p=0.28, n=9$ ) and artificial cerebrospinal fluid (ACSF)-applied control neurons $(n=7)$. (b) Cumulative probability density plot shows the overlap between tianeptine and control neurons, indicating the absence of tianeptine effects ( $p>0.6)$ on AMPA EPSCs. (c, top) Representative NMDAR-EPSCs for baseline, tianeptine application, and washout. (Bottom) Time-course plot for NMDA currents indicate a transient initial rise $(107 \pm 3 \%, n=14, p=0.007)$, but was similar to pre-drug baseline during tianeptine application ( $94 \pm 3 \%$, $p=0.58)$. However, by the end of washout, NMDA-EPSCs declined in tianeptine-treated cells $(85 \pm 5 \%, p=0.002)$ and not in control cells $(105 \pm 3 \%, n=13, p>0.2)$. (d) The significant $(* p<0.03)$ leftward shift in the cumulative probability plot highlights the decline of NMDA-EPSCs during washout in tianeptine-treated cells. The epoch of averaged sample traces and cumulative plots are indicated by the position of the respective markers on the time-course plot. Significances were obtained by comparing tianeptine and control neurons at similar time points (as indicated by the markers) using two-sample Kolmogorov-Smirnov test. Error bars represent standard error of mean (SEM).

tianeptine. Subsequent washout for 30 min dampened the tianeptine-induced potentiation as indicated by the leftward shift compared with that seen at the end of drug application. No such rightward shift in AMPA-EPSCs was seen after ACSF application.

Next, the same experimental design was used to assess the impact of $50 \mu \mathrm{M}$ tianeptine on NMDAR-EPSCs at Schaffer collateral inputs to CA1 pyramidal cells. Similar to AMPAEPSCs, bath application of tianeptine elicited a significant increase in the NMDA-EPSC peak amplitude. As shown in the time-course plot (Figure 1c, bottom), at the end of the drug application, the average NMDA-EPSC peak amplitude more than doubled relative to pre-drug baseline values. This potentiating effect of tianeptine persisted even after the 30min washout with ACSF (Figure 1c). This residual enhancing effect on NMDA-EPSCs was more pronounced than AMPA-EPSCs. The cumulative probability distribution for NMDA-EPSCs recorded from all tianeptine-treated cells exhibited a significant rightward shift that persisted even after drug washout, albeit at a lower level (Figure 1d).
Further, this enhancing effect of tianeptine was not caused by a shift in the reversal potential of NMDA-EPSCs (Supplementary Figure 1). Control experiments with ACSF treatment did not exhibit any of the above effects.

\section{Tianeptine Selectively Reduces Synaptic Currents Mediated by NMDA, but not AMPA, Receptors in the LA}

Our recordings in hippocampal slices show that tianeptine potentiates both AMPA and NMDA currents. Would these synaptic currents respond to tianeptine in a similar manner in the LA? To answer this question, we recorded EPSCs evoked by stimulation of thalamic afferents to principal neurons in the dorsal LA (Figure 2a; Supplementary Information). Unlike CA1 neurons, there was no effect on AMPA-EPSCs in LA neurons at any point during the 15-min bath application of tianeptine $(50 \mu \mathrm{M}$; Figure $2 \mathrm{a})$. Peak values of AMPA-EPSCs at the end of drug application were indistinguishable from pre-drug baseline values or control cells with ACSF application (Figure 2a, bottom). Similar 


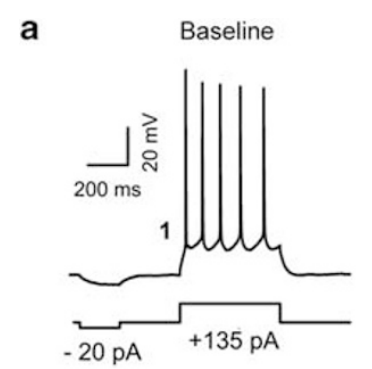

b

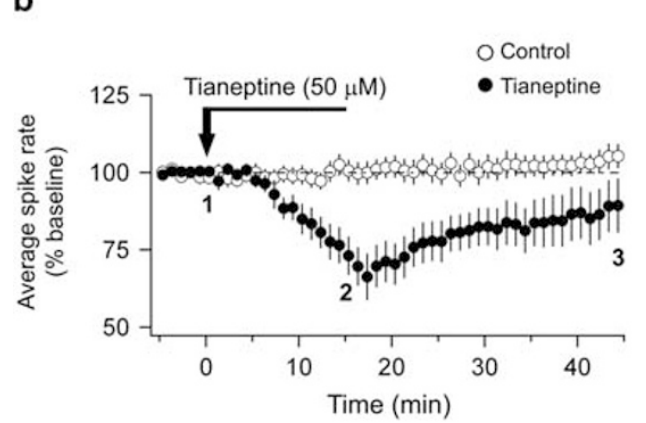

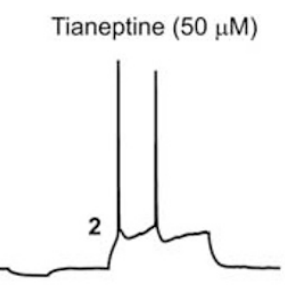
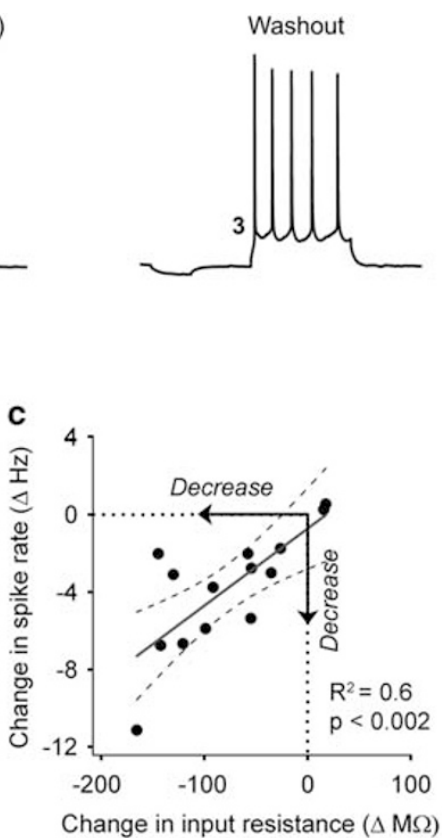

Figure 3 Tianeptine reduces intrinsic excitability in lateral amygdala (LA) projection neurons. (a) Representative traces of action potentials induced via depolarizing current injections (inset) during baseline, tianeptine application, and washout. (b) The time-course plot illustrates the decrease in \% normalized spike rate after tianeptine application $(8 \mathrm{I} \pm 5 \%, n=13, p=0.005)$, which was partially restored by washout $(88 \pm 8, p=0.082)$ compared to the steady responses of artificial cerebrospinal fluid (ACSF)-applied control cells $(n=I I)$. (c) As indicated in the correlation plot, there is a direct relationship between the change in spike rate and input resistance in tianeptine-applied neurons $\left(R^{2}=0.6, n=14, p<0.002\right.$, Pearson's correlation). A least-square fit line (gray) is shown along with 95\% confidence (dotted lines) bands. Error bars represent standard error of mean (SEM).

results were obtained for AMPA-EPSCs in tianeptinetreated cells at the end of 30-min washout. This lack of effect, at the end of drug application as well as washout, is confirmed in the overlapping cumulative probability distribution plots for the tianeptine, washout, and control cells (Figure 2b).

Having found no effect of tianeptine on AMPA-EPSCs, we shifted our focus to NMDA-EPSCs. Tianeptine $(50 \mu \mathrm{M})$, apart from a transient and small initial increase, did not cause any persistent and significant change in NMDAEPSCs at the end of the 15-min bath application. However, the effects of tianeptine became evident later when the drug was washed out with ACSF - there was a delayed reduction in NMDA-EPSC amplitude approximately $20 \mathrm{~min}$ after the drug was first bath applied (Figure 2c). Even after the 30min washout, this significant reduction in NMDA-EPSC amplitudes persisted. This reduction was not caused by a shift in the reversal potential of NMDA-EPSCs (Supplementary Figure 2). Although this decrease was seen with a time delay after application of tianeptine, the effect was nonetheless specific to the drug because no such reduction was elicited by ACSF in control neurons (Figure 2c). These effects of tianeptine are also evident in the cumulative probability distribution, where the slight leftward shift (ie, reduced amplitude) seen at the end of tianeptine application stabilizes into a significant leftward shift approximately $30 \mathrm{~min}$ after the end of tianeptine treatment. Taken together, these results indicate that tianeptine selectively reduces NMDAR-EPSCs in the LA without having any effect on AMPA-EPSCs. Notably, both of these effects are in clear contrast to the synaptic effects elicited by tianeptine in the hippocampus.

\section{Tianeptine Reduces Action Potential Firing in LA Neurons}

Although changes in synaptic efficacy have been the primary focus of the past studies on the cellular basis of experience or activity-induced neural plasticity, more recent work has highlighted the importance of changes in intrinsic excitability of neurons in such processes (McKay et al, 2009; Santini et al, 2008; Zhang and Linden, 2003). Further, modulation of spike firing in LA neurons plays a pivotal role in neuronal signaling related to emotional information processing in the amygdala (Maren and Quirk, 2004). Therefore, we also examined if tianeptine affects firing of action potentials in LA neurons. Whole-cell current-clamp recordings were obtained from LA projection neurons that exhibited spike frequency adaptation upon depolarizing current injections (Supplementary Information). Bath application of tianeptine $(50 \mu \mathrm{M}, 15 \mathrm{~min})$ leads to a steady decrease in action potential firing when compared to baseline activity (Figure 3a). However, after a 30-min washout this reduction in firing rates recovered partially to levels closer to pre-drug baseline values. In control experiments, treatment with ACSF alone had no effect on spiking.

In light of this modulation of neuronal excitability by tianeptine, we also assessed its effects on active and passive membrane properties of LA pyramidal neurons (Supplementary Table 1; Supplementary Information). Further, treatment with tianeptine had no effects on hyperpolarization-activated currents $\left(I_{\mathrm{h}}\right)$, after-hyperpolarizing potential, and GABAergic miniature inhibitory post-synaptic currents (see Supplementary Figures 3-6). However, the same in vitro tianeptine treatment led to a significant reduction 

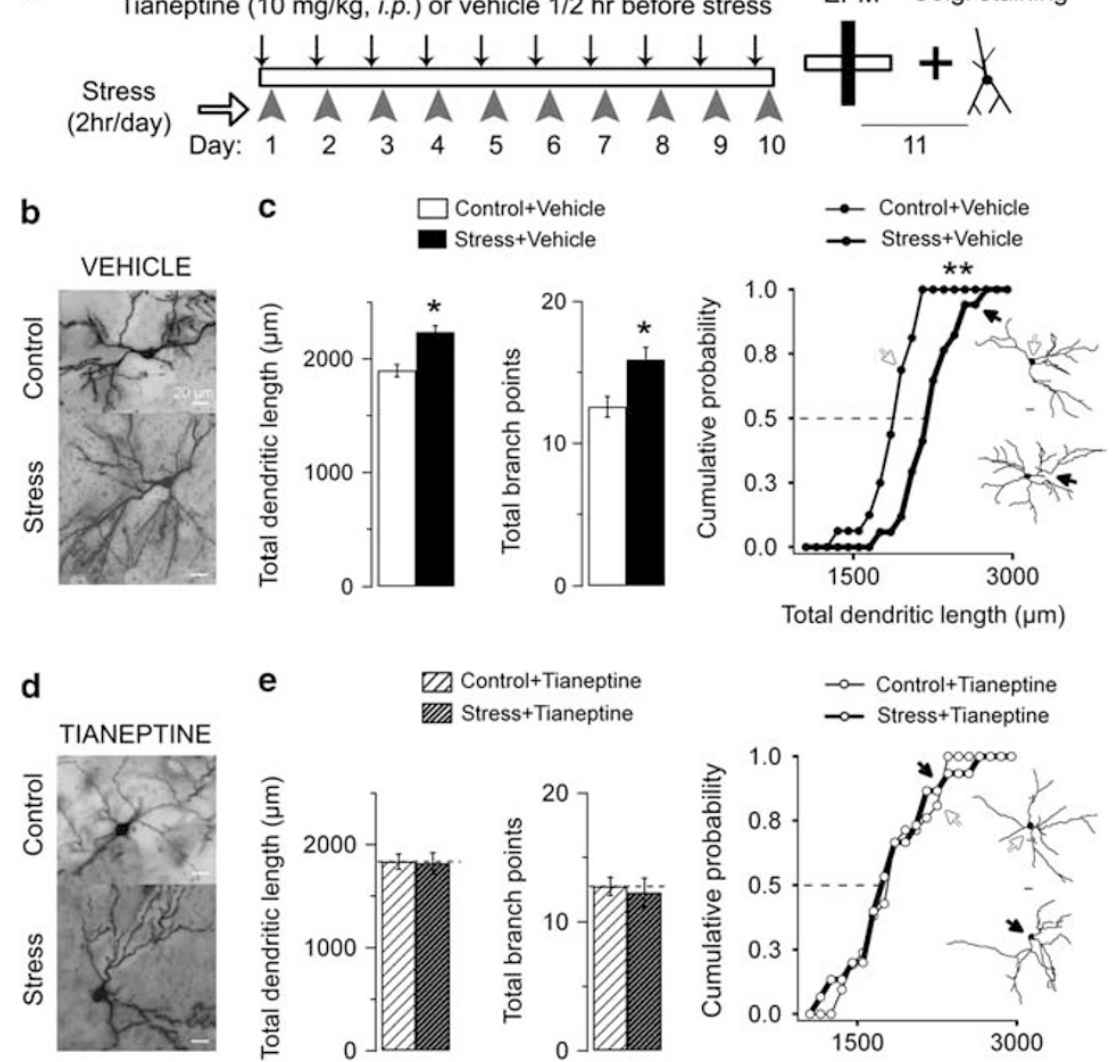

Z7 Control+Tianeptine
WTA Stress+Tianeptine

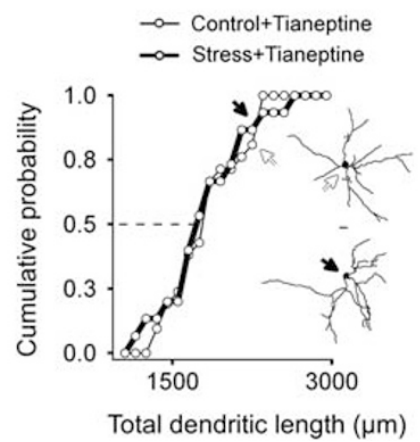

Figure 4 Tianeptine blocks stress-induced dendritic growth in the basolateral amygdala (BLA) spiny neurons. (a) As illustrated in the schematic of the experimental procedure, animals received daily injections of either tianeptine ( $10 \mathrm{mg} / \mathrm{kg}$; intraperitoneally (i.p.)) or vehicle $30 \mathrm{~min}$ before stress $(2 \mathrm{~h}$ per day) for 10 consecutive days. On the IIth day, all animals were killed after testing for anxiety and the brains were processed for rapid Golgi staining. (b) Representative images of Golgi-impregnated BLA principal neurons from vehicle-treated control (top) and stress (bottom) animals (scale bar: $20 \mu \mathrm{m}$ ). (c, left and middle) Mean total dendritic length (control + vehicle: I $898 \pm 53 \mu \mathrm{m}, n=16$; stress + vehicle: $2235 \pm 58 \mu \mathrm{m}, n=17$; ${ }^{*} p<0.00$ I, * $p<0.0$ I3) and branch points (control + vehicle: $12.6 \pm 0.7$; stress + vehicle: $15.9 \pm 0.9,{ }^{\#} p<0.014,{ }^{*} p<0.05$ ) of BLA neurons were significantly increased after stress when compared to vehicle-injected control animals. (Right) Cumulative probability density of total dendritic length in neurons from stressed animals was rightward shifted in comparison to their unstressed counterparts. (Inset) Representative neuron traces, sampled at specific locations (arrows) of the cumulative plot. (d) Representative images of Golgi-impregnated BLA principal neurons from tianeptine-treated control (top) and stress (bottom) animals (scale bar: $20 \mu \mathrm{m}$ ). (e, left and middle) Mean total dendritic length (control + tianeptine: $1834 \pm 74 \mu \mathrm{m}, n=21$; stress + tianeptine: $1818 \pm 103 \mu \mathrm{m}, n=15$, * $p>0.9)$ and branch points (control + tianeptine: $12.8 \pm 0.7$; stress + tianeptine: $12.3 \pm 1.1$; * $>0.9$ ) of BLA neurons were similar between tianeptine-treated stress and control animals. (Right) Cumulative plot illustrates the lack of effect of stress on BLA neurons in animals that received chronic tianeptine administration. (Inset) Representative neuron traces, sampled at specific locations (arrows) of the cumulative plot. Error bars represent SEM. The scale bars equal $20 \mu \mathrm{m}$; ' $n$ ': number of neurons. " $p$, One-way analysis of variance (ANOVA); *p, post-hoc Tukey's honestly significant difference (HSD); and *** $<0.01$, two-sample Kolmogorov-Smirnov test.

in input resistance at the end of $15 \mathrm{~min}$ of drug application $(-16 \%$ relative to pre-drug baseline; $n=14, p=0.004)$. We also find a significant correlation between the changes in firing rates and input resistance at the end of tianeptine treatment (Figure 3c). However, changes in these two measures following bath application of ACSF alone were not correlated $(p=0.5)$ in control cells. Thus, the reduction in spike firing elicited by tianeptine may be mediated, at least in part, via decrease in input resistance. However, the underlying mechanisms and the role of specific ion channels in this process await further investigation.

\section{Tianeptine Blocks Chronic Stress-Induced Dendritic Hypertrophy in the BLA Neurons}

Do the in vitro electrophysiological effects of tianeptine described above have any functional consequences or potential benefits in the intact animal? To address this question, we took into consideration two important issues. First, the impact of this drug on glutamatergic synaptic currents differs between the hippocampus and amygdala. Second, this region-specific difference is significant in light of earlier studies reporting opposite effects of chronic stress on these two brain areas (Kavushansky et al, 2006; Vouimba et al, 2004, 2006; Vyas et al, 2002). Repeated stress causes hippocampal dendritic atrophy, which in turn depends on NMDA receptors. Importantly, tianeptine prevents stressinduced dendritic atrophy in the hippocampus. However, chronic stress elicits the opposite effect-dendritic hypertrophy - in the BLA. As tianeptine triggers opposing effects on NMDA-EPSCs, would tianeptine also prevent the contrasting morphological effect of chronic stress in the BLA?

To address this question, we utilized a rodent model of CIS ( $2 \mathrm{~h}$ per day, 10 days) that is reported to cause dendritic 
growth in BLA principal neurons (Vyas et al, 2002). Stressed animals were treated with either vehicle or tianeptine $30 \mathrm{~min}$ before the start of the daily 2 -h session of immobilization (Figure 4a), and these were compared with unstressed control animals subjected to the same schedule of vehicle or tianeptine treatment. Dendritic arborization of Golgi-stained BLA principal neurons from vehicle-treated animals subjected to chronic stress were compared with their unstressed counterparts. There was a significant increase in total dendritic length and the number of branch points (Figure $4 \mathrm{~b}$ ) caused by chronic stress in vehicletreated animals. Moreover, there was a significant $(p<0.003)$ rightward shift in the cumulative frequency plot for the stress + vehicle neurons compared to control+ vehicle neurons, indicating that the observed dendritic hypertrophy was present across a wide range of total dendritic length values for all the BLA neurons analyzed (Figure 4b). Taken together, these data from vehicleinjected animals are consistent with previous reports on stress-induced dendritic growth in BLA principal neurons (Vyas et al, 2002, 2004).

By contrast, in tianeptine-treated animals $(10 \mathrm{mg} / \mathrm{kg}$, intraperitoneally; injected $30 \mathrm{~min}$ before stress), the same chronic stress failed to elicit dendritic hypertrophy in BLA principal neurons (Figure 4c). Tianeptine blocked stressinduced increases in both total dendritic length and number of branch points. Furthermore, the close overlap in the cumulative frequency plots for the two groups of BLA neurons $(p>0.9$; Figure $4 c)$ is in stark contrast to the separation between the corresponding plots from the vehicle-treated animals (Figure $4 \mathrm{~b}$ ). Thus, in vivo treatment with tianeptine is indeed capable of preventing BLA dendritic growth caused by chronic stress.

\section{Tianeptine Prevents Chronic Stress-Induced Facilitation of Anxiety-Like Behavior}

There is evidence linking stress-induced dendritic remodeling in the amygdala and facilitation of anxiety-like behavior (Adamec et al, 2012; Hill et al, 2011; Mitra et al, 2009; Vyas et al, 2002). As tianeptine is effective in blocking amygdalar dendritic hypertrophy triggered by chronic stress, we next investigated whether it is also able to prevent the anxiogenic effects of stress. Therefore, animals from all four experimental groups were tested on the elevated plus maze $24 \mathrm{~h}$ after the end of the same 10-day chronic stress protocol (Figure 4a). There was a significant main effect of stress in both percentage open-arm time and entries, indicating that vehicle-treated animals exposed to chronic stress exhibited significantly higher anxiety-like behavior in the plus maze compared to unstressed animals (Figure 5a). Thus, in agreement with previous reports, chronic stress enhanced anxiety in vehicle-injected animals.

In striking contrast, in tianeptine-treated animals stress did not affect either the percentage of open-arm time or entries (Figure 5b). The effect of tianeptine in preventing stress-induced anxiety was further validated from a significant interaction between stress and drug both in percentage entries $\left(\mathrm{F}_{(1,38)}=4.7, p<0.05\right)$ and time spent in the open arms $\left(\mathrm{F}_{(1,38)}=5.2, p<0.05\right)$. Further, the average closed-arm entries (control + vehicle: $4.1 \pm 0.5$; stress + vehicle: $4.1 \pm 0.4$; control + tianeptine: $4.3 \pm 0.5$; stress +
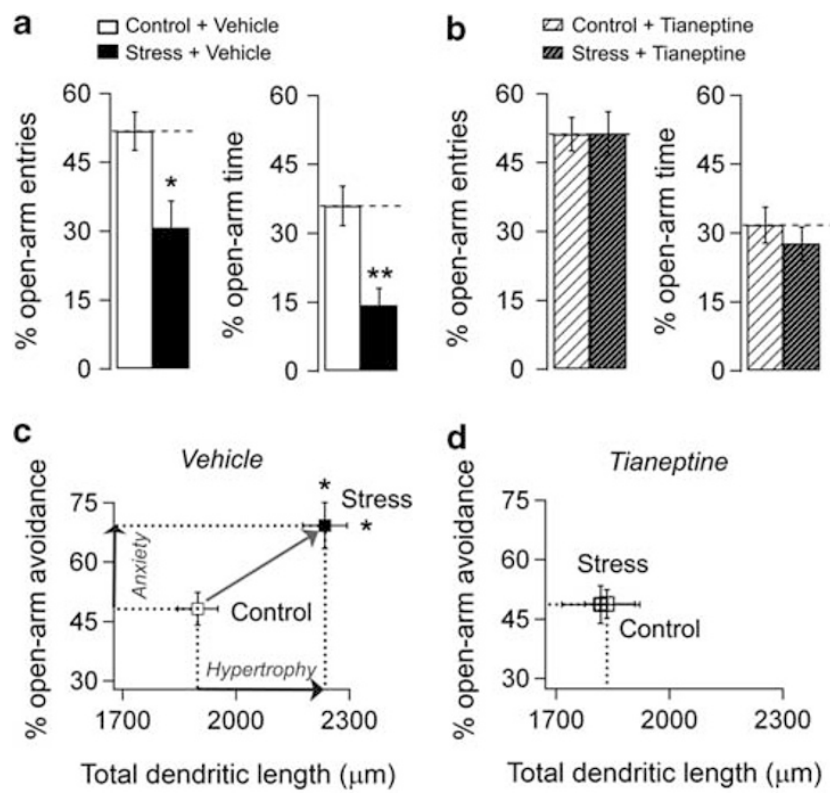

Figure 5 Tianeptine prevents stress-induced facilitation of anxiety-like behavior. (a) Averaged values of normalized (\% total) open-arm entries (control + vehicle: $\quad 52 \pm 4, \quad n=9 ; \quad$ stress + vehicle: $\quad 31 \pm 6, \quad n=12$; $\left.F_{(1,38)}=4.6, p<0.05\right)$ and time (control + vehicle: $36 \pm 4 \%$; stress + vehicle: $\left.14 \pm 4 ; F_{(1,38)}=10.9, p<0.01\right)$ were different between vehicle-injected stress and control animals. (b) Averaged values of open-arm entries (control + tianeptine: $51 \pm 4, n=10$; stress + tianeptine: $51 \pm 5, n=11$, $p>0.9$ ) and time (control + tianeptine: $32 \pm 4, n=10$; stress + tianeptine: $28 \pm 4, n=11, p>0.9)$ were not different between tianeptine-administered (10 mg/kg; intraperitoneally (i.p.)) stressed and control animals. (c) Summary of behavioral and morphological effects for vehicle-injected animals showing an increase in both percentage open-arm avoidance ( $100 \%$, percentage of open-arm entries; vertical arrow, Anxiety) and total dendritic length (horizontal arrow, Hypertrophy). (d) Summary of behavioral and morphological effects for tianeptine-injected animals indicating the lack of stress-induced facilitation of anxiety as well as dendritic hypertrophy. Error bars represent standard error of mean (SEM); $n$ : animals per group. Statistical significances ( $* p<0.5$, $* * p<0.0$ I) were computed from one-way analysis of variance (ANOVA) and Tukey's posthoc test.

tianeptine: $4.5 \pm 0.6)$ were similar across the groups $(p>0.9$, one-way ANOVA), demonstrating the similarity in locomotor activity between the four groups.

Figure $5 \mathrm{c}$ and $\mathrm{d}$ summarizes the combined results of stress and tianeptine effects at both the cellular and behavioral levels. In vehicle-treated animals, the total dendritic length of BLA neurons (Hypertrophy, rightward arrow, Figure $5 \mathrm{c}$ ), as well as open-arm avoidance in the plus maze (Anxiety, upward arrow, Figure 5c), is significantly greater in stressed animals compared to unstressed controls. However, treatment with tianeptine prevents the increase in anxiety and dendritic growth such that the control and stressed groups are indistinguishable (overlapping points in Figure 5d).

\section{DISCUSSION}

Here we report that the same antidepressant is capable of triggering divergent patterns of electrophysiological changes that are well-suited for countering the contrasting effects of chronic stress in the hippocampus and amygdala. To investigate the functional consequences of the unique 
physiological actions of tianeptine in the amygdala, we were guided by earlier reports on the efficacy of tianeptine in preventing hippocampal dendritic atrophy and impaired learning and memory caused by stress (Conrad et al, 1996; McEwen et al, 1997). As the effects of chronic stress on the amygdala are quite different from the hippocampus (Kavushansky et al, 2006; Roozendaal et al, 2009; Vouimba et al, 2004), we tested if the contrasting in vitro effects of tianeptine in the two areas also lead to functional benefits in the intact animal against stress. Indeed, systemic administration of tianeptine prevents stress-induced facilitation of anxiety-like behavior and BLA dendritic growth. Thus, despite the contrasting effects of stress on the hippocampus $v s$ amygdala, tianeptine appears to be effective in countering both. Taken together, our findings add to a growing body of evidence on cellular mechanisms in the amygdala that are different from the hippocampus, not only with respect to their response to stress, but also to potential therapeutic interventions against stress.

Rodent studies have demonstrated the robust antidepressant properties of tianeptine, especially in countering the adverse impact of stress at multiple levels of neural organization (Czeh et al, 2001; Kuroda and McEwen, 1998). These results are paralleled by clinical data demonstrating the efficacy of tianeptine in treating a range of cognitive and affective symptoms of depression in humans (Costa e Silva, 2004). These pre-clinical and clinical observations highlight the need to look beyond the traditional focus on the serotonergic system in depression (Javitt, 2004; Paul and Skolnick, 2003; Rainnie, 1999; Sanacora et al, 2003). Specifically, there is a growing consensus that tianeptine achieves its neuroprotective effects in corticolimbic structures, and thereby, its clinical efficacy, by acting directly on glutamatergic synapses (McEwen et al, 2010). Early indications of the atypical mode of action of tianeptine came from pharmacological membrane binding assays showing that this compound does not bind to serotonergic receptors or transporters (Kato and Weitsch, 1988; Pineyro et al, 1995). More recently, both acute and long-term administration of tianeptine have been shown not to affect extracellular levels of serotonin (Malagie et al, 2000). Compared to the lack of any direct role for serotonin in the antidepressant action of tianeptine, there is evidence for its actions on glutamatergic synapses. Of direct relevance to our findings is an earlier report that tianeptine prevents stress-induced re-scaling of the ratio of NMDA and AMPA EPSCs in hippocampal slices (Kole et al, 2002). Systemic treatment with tianeptine also amplified the slope of the inputoutput curve for EPSCs in slices from stressed and unstressed rats. Further, in vitro application of tianeptine rapidly increased the amplitudes of NMDA- and AMPAEPSCs in area CA3, similar to what we find in area CA1. Interestingly, in the same study, tianeptine-induced alteration in the phosphorylation state of glutamate receptors was implicated in the enhancement of EPSCs. This is consistent with a recent molecular analysis showing that tianeptine treatment increased phosphorylation of the Ser831 site on the GluR1 subunit of AMPARs in the hippocampus (Svenningsson et al, 2007). As phosphorylation of GluR1 subunits is expected to potentiate AMPAR function, our results on tianeptine-induced enhancement of AMPA-EPSCs provide a direct electrophysiological validation of this prediction.

Modulation of the glutamatergic system by tianeptine also provides a point of convergence for the compound to act upon synaptic plasticity mechanisms underlying hippocampal learning and memory. Tianeptine is known to reverse stress-induced inhibition of LTP within the hippocampus (Shakesby et al, 2002; Vouimba et al, 2006), and its projections to the prefrontal cortex (Rocher et al, 2004). Further, Diamond and co-workers have reported that tianeptine also enhances primed-burst potentiation in area CA1 even in the absence of stress (Diamond et al, 2004; Vouimba et al, 2006). These reports highlight key features of the effects of tianeptine in the hippocampus - it facilitates basal synaptic transmission and LTP in unstressed animals, and also prevents stress-induced inhibition of LTP. Our data suggest mechanisms by which both of these benefits may be achieved. First, tianeptine-induced increase in NMDA-EPSCs provides an ideal synaptic substrate for facilitating LTP, thereby countering its suppression by stress. Second, as the expression of LTP is mediated through a strengthening of AMPAR signaling, the tianeptine-induced increase in AMPA-EPSCs by itself mimics an LTP-like effect in the hippocampus. This ability of tianeptine to restore and protect normal hippocampal plasticity explains its efficacy against stress-induced impairment of spatial memory (Campbell et al, 2008; Conrad et al, 1996; Luine et al, 1994; Morris et al, 2001).

While stress impairs hippocampal function across levels, it triggers opposite effects in the amygdala - from enhanced anxiety and fear at the behavioral level to dendritic growth and enhanced LTP at the cellular level (Roozendaal et al, 2009). Thus, a primary goal of this study was to examine if tianeptine also differs in its effects on these two brain areas. We find that tianeptine modulates the same subtypes of glutamate receptors in the amygdala as in the hippocampus, but in a strikingly different manner. Are the contrasting effects of tianeptine in the amygdala consistent with its reported modulation of amygdalar plasticity and its functional consequences? NMDARs play a central role in amygdalar LTP and fear memory formation, and chronic tianeptine treatment before acquisition of fear conditioning inhibits subsequent recall of the fear memory (Burghardt et al, 2004). Further, exposure to stress facilitates various forms of classical fear conditioning and anxiety in rats (Shors and Mathew, 1998; Vyas et al, 2004). Conversely, local infusion of NMDAR antagonists into the BLA prevents these facilitatory effects of stress (Shors and Mathew, 1998). These observations are consistent with a persistent reduction in NMDA-EPSCs caused by tianeptine in the amygdala. Moreover, while past research has focused on synaptic mechanisms, we show that tianeptine also modulates nonsynaptic measures of intrinsic excitability by lowering action potential firing rates. While our results suggest that reduced input resistance may contribute to the lower firing rates, more detailed analysis will be needed to examine a potential role for other mechanism including ion channels activated by G-protein-coupled receptors. As fear conditioning enhances spike firing evoked by the conditional stimulus in LA neurons (Maren and Quirk, 2004), tianeptine-induced decrease in spiking rates could also contribute to the reduction in fear conditioning (Burghardt 
et al, 2004). Consistent with its efficacy in reducing fear conditioning, we find that chronic treatment with tianeptine prevents the anxiogenic effects of chronic stress; this is in agreement with its reported prevention of the anxiogenic effects of benzodiazepine and alcohol withdrawal (File et al, 1993). In this study, our choice of the elevated plus maze for measuring the effects of tianeptine on anxiety-like behavior was guided primarily by earlier studies that had established a link between stress-induced reduction in open-arm exploration on the plus maze and dendritic hypertrophy in the BLA. Future studies will be needed to extend such analysis to commonly used animal models of depression and anxiety.

Our behavioral data on the anxiolytic effect of tianeptine led us to an important question at the cellular level: since previous reports have established a link between chronic stress-induced facilitation of anxiety and dendritic growth in the amygdala, would the latter also be suppressed by tianeptine? We found this to be the case. Although little is known about mechanisms underlying stress-induced structural plasticity in the amygdala, there is evidence for involvement of NMDARs in stress-induced hippocampal dendritic remodeling (McEwen, 1999), as well as dendritic development in other model systems (Cline, 2001). Tianeptine-induced decrease in NMDA-EPSCs reported here suggests a similar mechanism in the amygdala. Further, Reznikov et al (2007) have shown that extracellular glutamate levels are enhanced by stress in the amygdala, and this too is reversed by tianeptine. Thus, excess glutamate and its electrophysiological actions on receptors that trigger plasticity are both targeted by tianeptine in the amygdala. Tianeptine has also been shown to modulate brain-derived neurotrophic factor expression in the rat amygdala (Reagan et al, 2007). The potential therapeutic relevance of these glutamatergic mechanisms is reinforced by clinical data indicating the efficacy of the NMDAR antagonist ketamine in depressed patients (Berman et al, 2000). Therefore, modulation of plasticity at glutamatergic synapses (Zarate et al, 2003), using compounds such as tianeptine, is likely to provide a useful framework for developing more effective antidepressants in the future.

\section{ACKNOWLEDGEMENTS}

SC is the recipient of an International Senior Research Fellowship from the Wellcome Trust, UK. AG was supported by a research grant from Hikal, India. The authors thank Serdia, India for providing reagents.

\section{DISCLOSURE}

All authors reported no biomedical financial interests or potential conflicts of interest.

\section{REFERENCES}

Adamec R, Hebert M, Blundell J, Mervis RF (2012). Dendritic morphology of amygdala and hippocampal neurons in more and less predator stress responsive rats and more and less spontaneously anxious handled controls. Behav Brain Res 226: 133-146.
Arborelius L, Owens MJ, Plotsky PM, Nemeroff CB (1999). The role of corticotropin-releasing factor in depression and anxiety disorders. J Endocrinol 160: 1-12.

Berman RM, Cappiello A, Anand A, Oren DA, Heninger GR, Charney DS et al (2000). Antidepressant effects of ketamine in depressed patients. Biol Psychiatry 47: 351-354.

Bremner JD, Narayan M, Anderson ER, Staib LH, Miller HL, Charney DS (2000). Hippocampal volume reduction in major depression. Am J Psychiatry 157: 115-118.

Burghardt NS, Sullivan GM, McEwen BS, Gorman JM, LeDoux JE (2004). The selective serotonin reuptake inhibitor citalopram increases fear after acute treatment but reduces fear with chronic treatment: a comparison with tianeptine. Biol Psychiatry 55: 1171-1178.

Campbell AM, Park CR, Zoladz PR, Munoz C, Fleshner M, Diamond DM (2008). Pre-training administration of tianeptine, but not propranolol, protects hippocampus-dependent memory from being impaired by predator stress. Eur Neuropsychopharmacol 18: 87-98.

Cline HT (2001). Dendritic arbor development and synaptogenesis. Curr Opin Neurobiol 11: 118-126.

Conrad CD, Galea LA, Kuroda Y, McEwen BS (1996). Chronic stress impairs rat spatial memory on the Y maze, and this effect is blocked by tianeptine pretreatment. Behav Neurosci 110: 1321-1334.

Costa e Silva JA (2004). From restoration of neuroplasticity to the treatment of depression: clinical experience. Eur Neuropsychopharmacol 14(Suppl 5): S511-S521.

Czeh B, Michaelis T, Watanabe T, Frahm J, de Biurrun G, van Kampen $M$ et al (2001). Stress-induced changes in cerebral metabolites, hippocampal volume, and cell proliferation are prevented by antidepressant treatment with tianeptine. Proc Natl Acad Sci USA 98: 12796-12801.

Diamond DM, Campbell A, Park CR, Vouimba RM (2004). Preclinical research on stress, memory, and the brain in the development of pharmacotherapy for depression. Eur Neuropsychopharmacol 14(Suppl 5): S491-S495.

File SE, Andrews N, al Farhan M (1993). Anxiogenic responses of rats on withdrawal from chronic ethanol treatment: effects of tianeptine. Alcohol Alcohol 28: 281-286.

Frodl T, Meisenzahl EM, Zetzsche T, Born C, Groll C, Jager M et al (2002). Hippocampal changes in patients with a first episode of major depression. Am J Psychiatry 159: 1112-1118.

Hill MN, Hillard CJ, McEwen BS (2011). Alterations in corticolimbic dendritic morphology and emotional behavior in cannabinoid CB1 receptor-deficient mice parallel the effects of chronic stress. Cereb Cortex 21: 2056-2064.

Javitt DC (2004). Glutamate as a therapeutic target in psychiatric disorders. Mol Psychiatry 9: 984-997, 979.

Kato G, Weitsch AF (1988). Neurochemical profile of tianeptine, a new antidepressant drug. Clin Neuropharmacol 11(Suppl 2): S43-S50.

Kavushansky A, Vouimba RM, Cohen H, Richter-Levin G (2006). Activity and plasticity in the CA1, the dentate gyrus, and the amygdala following controllable vs. uncontrollable water stress. Hippocampus 16: 35-42.

Kim SJ, Park SH, Choi SH, Moon BH, Lee KJ, Kang SW et al (2006). Effects of repeated tianeptine treatment on CRF mRNA expression in non-stressed and chronic mild stress-exposed rats. Neuropharmacology 50: 824-833.

Kole MH, Swan L, Fuchs E (2002). The antidepressant tianeptine persistently modulates glutamate receptor currents of the hippocampal CA3 commissural associational synapse in chronically stressed rats. Eur J Neurosci 16: 807-816.

Kuroda Y, McEwen BS (1998). Effect of chronic restraint stress and tianeptine on growth factors, growth-associated protein-43 and microtubule-associated protein 2 mRNA expression in the rat hippocampus. Brain Res Mol Brain Res 59: 35-39. 
Luine V, Villegas M, Martinez C, McEwen BS (1994). Repeated stress causes reversible impairments of spatial memory performance. Brain Res 639: 167-170.

Magarinos AM, Deslandes A, McEwen BS (1999). Effects of antidepressants and benzodiazepine treatments on the dendritic structure of CA3 pyramidal neurons after chronic stress. Eur J Pharmacol 371: 113-122.

Malagie I, Deslandes A, Gardier AM (2000). Effects of acute and chronic tianeptine administration on serotonin outflow in rats: comparison with paroxetine by using in vivo microdialysis. Eur J Pharmacol 403: 55-65.

Maren S, Quirk GJ (2004). Neuronal signalling of fear memory. Nat Rev Neurosci 5: 844-852.

McEwen BS (1999). Stress and hippocampal plasticity. Annu Rev Neurosci 22: 105-122.

McEwen BS, Chattarji S, Diamond DM, Jay TM, Reagan LP, Svenningsson $\mathrm{P}$ et al (2010). The neurobiological properties of tianeptine (Stablon): from monoamine hypothesis to glutamatergic modulation. Mol Psychiatry 15: 237-249.

McEwen BS, Conrad CD, Kuroda Y, Frankfurt M, Magarinos AM, McKittrick C (1997). Prevention of stress-induced morphological and cognitive consequences. Eur Neuropsychopharmacol 7(Suppl 3): S323-S328.

McEwen BS, Magarinos AM, Reagan LP (2002). Structural plasticity and tianeptine: cellular and molecular targets. Eur Psychiatry 17(Suppl 3): 318-330.

McKay BM, Matthews EA, Oliveira FA, Disterhoft JF (2009). Intrinsic neuronal excitability is reversibly altered by a single experience in fear conditioning. J Neurophysiol 102: 2763-2770.

Mitra R, Adamec R, Sapolsky R (2009). Resilience against predator stress and dendritic morphology of amygdala neurons. Behav Brain Res 205: 535-543.

Morris RG, Kelly S, Burney D, Anthony T, Boyer PA, Spedding M (2001). Tianeptine and its enantiomers: effects on spatial memory in rats with medial septum lesions. Neuropharmacology 41: 272-281.

Nestler EJ, Barrot M, DiLeone RJ, Eisch AJ, Gold SJ, Monteggia LM (2002). Neurobiology of depression. Neuron 34: 13-25.

Paul IA, Skolnick P (2003). Glutamate and depression: clinical and preclinical studies. Ann N Y Acad Sci 1003: 250-272.

Pineyro G, Deveault L, de Montigny C, Blier P (1995). Effect of prolonged administration of tianeptine on 5-HT neurotransmission: an electrophysiological study in the rat hippocampus and dorsal raphe. Naunyn Schmiedebergs Arch Pharmacol 351: 119-125.

Qi H, Mailliet F, Spedding M, Rocher C, Zhang X, Delagrange P et al (2009). Antidepressants reverse the attenuation of the neurotrophic MEK/MAPK cascade in frontal cortex by elevated platform stress; reversal of effects on LTP is associated with GluA1 phosphorylation. Neuropharmacology 56: 37-46.

Rainnie DG (1999). Serotonergic modulation of neurotransmission in the rat basolateral amygdala. J Neurophysiol 82: 69-85.

Ramkumar K, Srikumar BN, Shankaranarayana Rao BS, Raju TR (2008). Self-stimulation rewarding experience restores stressinduced CA3 dendritic atrophy, spatial memory deficits and alterations in the levels of neurotransmitters in the hippocampus. Neurochem Res 33: 1651-1662.

Reagan LP, Hendry RM, Reznikov LR, Piroli GG, Wood GE, McEwen BS et al (2007). Tianeptine increases brain-derived neurotrophic factor expression in the rat amygdala. Eur $J$ Pharmacol 565: 68-75.
Reagan LP, Rosell DR, Wood GE, Spedding M, Munoz C, Rothstein $J$ et al (2004). Chronic restraint stress up-regulates GLT-1 mRNA and protein expression in the rat hippocampus: reversal by tianeptine. Proc Natl Acad Sci USA 101: 2179-2184.

Reznikov LR, Grillo CA, Piroli GG, Pasumarthi RK, Reagan LP, Fadel J (2007). Acute stress-mediated increases in extracellular glutamate levels in the rat amygdala: differential effects of antidepressant treatment. Eur J Neurosci 25: 3109-3114.

Rocher C, Spedding M, Munoz C, Jay TM (2004). Acute stressinduced changes in hippocampal/prefrontal circuits in rats: effects of antidepressants. Cereb Cortex 14: 224-229.

Roozendaal B, McEwen BS, Chattarji S (2009). Stress, memory and the amygdala. Nat Rev Neurosci 10: 423-433.

Sachar EJ, Baron M (1979). The biology of affective disorders. Annu Rev Neurosci 2: 505-517.

Sanacora G, Rothman DL, Mason G, Krystal JH (2003). Clinical studies implementing glutamate neurotransmission in mood disorders. Ann N Y Acad Sci 1003: 292-308.

Santini E, Quirk GJ, Porter JT (2008). Fear conditioning and extinction differentially modify the intrinsic excitability of infralimbic neurons. J Neurosci 28: 4028-4036.

Shakesby AC, Anwyl R, Rowan MJ (2002). Overcoming the effects of stress on synaptic plasticity in the intact hippocampus: rapid actions of serotonergic and antidepressant agents. J Neurosci 22: 3638-3644.

Shors TJ, Mathew PR (1998). NMDA receptor antagonism in the lateral/basolateral but not central nucleus of the amygdala prevents the induction of facilitated learning in response to stress. Learn Mem 5: 220-230.

Suvrathan A, Hoeffer CA, Wong H, Klann E, Chattarji S (2010). Characterization and reversal of synaptic defects in the amygdala in a mouse model of fragile X syndrome. Proc Natl Acad Sci USA 107: 11591-11596.

Svenningsson P, Bateup H, Qi H, Takamiya K, Huganir RL, Spedding $\mathrm{M}$ et al (2007). Involvement of AMPA receptor phosphorylation in antidepressant actions with special reference to tianeptine. Eur J Neurosci 26: 3509-3517.

Vouimba RM, Munoz C, Diamond DM (2006). Differential effects of predator stress and the antidepressant tianeptine on physiological plasticity in the hippocampus and basolateral amygdala. Stress 9: 29-40.

Vouimba RM, Yaniv D, Diamond D, Richter-Levin G (2004). Effects of inescapable stress on LTP in the amygdala versus the dentate gyrus of freely behaving rats. Eur J Neurosci 19: 1887-1894.

Vyas A, Mitra R, Shankaranarayana Rao BS, Chattarji S (2002). Chronic stress induces contrasting patterns of dendritic remodeling in hippocampal and amygdaloid neurons. J Neurosci 22: 6810-6818.

Vyas A, Pillai AG, Chattarji S (2004). Recovery after chronic stress fails to reverse amygdaloid neuronal hypertrophy and enhanced anxiety-like behavior. Neuroscience 128: 667-673.

Watanabe Y, Gould E, Cameron HA, Daniels DC, McEwen BS (1992). Phenytoin prevents stress- and corticosterone-induced atrophy of CA3 pyramidal neurons. Hippocampus 2: 431-435.

Zarate Jr CA, Du J, Quiroz J, Gray NA, Denicoff KD, Singh J et al (2003). Regulation of cellular plasticity cascades in the pathophysiology and treatment of mood disorders: role of the glutamatergic system. Ann N Y Acad Sci 1003: 273-291.

Zhang W, Linden DJ (2003). The other side of the engram: experience-driven changes in neuronal intrinsic excitability. Nat Rev Neurosci 4: 885-900.

Supplementary Information accompanies the paper on the Neuropsychopharmacology website (http://www.nature.com/npp) 